\title{
Automated Rice Transplanting Operation Using GPS and FOG Sensors
}

\author{
Yoshisada NAGASAKA*, Ken TANIWAKI, Ryuji OTANI and Kazuto \\ SHIGETA
}

Department of Farm Mechanization, National Agriculture Research Center

(Tsukuba, Ibaraki, 305-8666 Japan)

\begin{abstract}
The objective of this study was to develop an automated rice transplanting system in paddy fields. It is necessary to determine the precise position and direction to control the vehicle automatically in paddy fields. In this paper, the position of the rice transplanter was determined by real-time kinematic GPS (RTKGPS). The inclination of the rice transplanter influenced the RTKGPS position data, including time delay because the GPS antenna was set at a $2 \mathrm{~m}$ height from the ground. The correction can be performed by measuring the roll, the pitch and the yaw angles of the rice transplanter. The yaw angle was measured using a fiber optic gyro (FOG) sensor and the roll and pitch angles were measured using an inclination-measuring apparatus consisting of 3 FOG sensors and 3 accelerometers. When the rice transplanter was driven automatically along the desired straight path, the deviation from the desired straight path was less than $10 \mathrm{~cm}$.
\end{abstract}

Discipline: Agricultural machinery

Additional key words: real-time kinematic GPS, rice transplanter, inclination-measuring apparatus

\section{Introduction}

Recently in Japan, though there has been a trend to consolidate and enlarge paddy fields, the number of farmers is decreasing. A more efficient operation system is needed because the workload of the farmers is increasing. If an automated operation system could be developed, one person could operate multiple machines. The objective of this study was to develop an automated operation system to enhance the efficiency of the operations. In order to perform automated operations, it is necessary to determine the position and direction of the vehicle in the program and to drive precisely. In recent studies, Yukumoto et al. ${ }^{6}$ (1995) used an optical wave range finder and magnetic azimuth sensor. Noguchi et al. ${ }^{5)}$ (1997) used an image processing and magnetic azimuth sensor. However, these techniques require equipment in the field and the precision of location is influenced by the weather because optical instruments were used. Inoue et al. ${ }^{2)}$ (1997) used differential GPS and FOG sensors. Elkaim et al. ${ }^{1)}$ (1997) used carrier phase differential GPS. They obtained good results for tillage, but the instruments lacked the requisite precision for rice transplanting. For the rice transplanting operation, the vehicles must be driven accurately because the inter-row spacing of rice plants is about $30 \mathrm{~cm}$ in Japan. However, unevenness of the ground and side slip of the wheel disturb the vehicle direction. Compared with driving on solid terrain, it is difficult to drive a vehicle along the straight desired path in paddy fields. The authors ${ }^{3)}$ used a RTKGPS with a 1 $\mathrm{cm}$ precision at $1 \mathrm{~Hz}$ data output to locate the position, but the time delay of the RTKGPS data output was long, and the authors could not obtain satisfactory results. The authors used a new RTKGPS system and the vehicle control method was improved ${ }^{4}$. The time delay was shortened and the precision of location was improved. In this paper, the authors describe the correction of the vehicle inclination, synchronization of the RTKGPS data, the yaw angle and the inclination data, the estimation of the real time deviation from the desired path and the vehicle control method.

\section{Materials and methods}

\section{1) Automated rice transplanting system}

This automated operation system consists of an RTKGPS reference station and a 5-row rice transplanter 


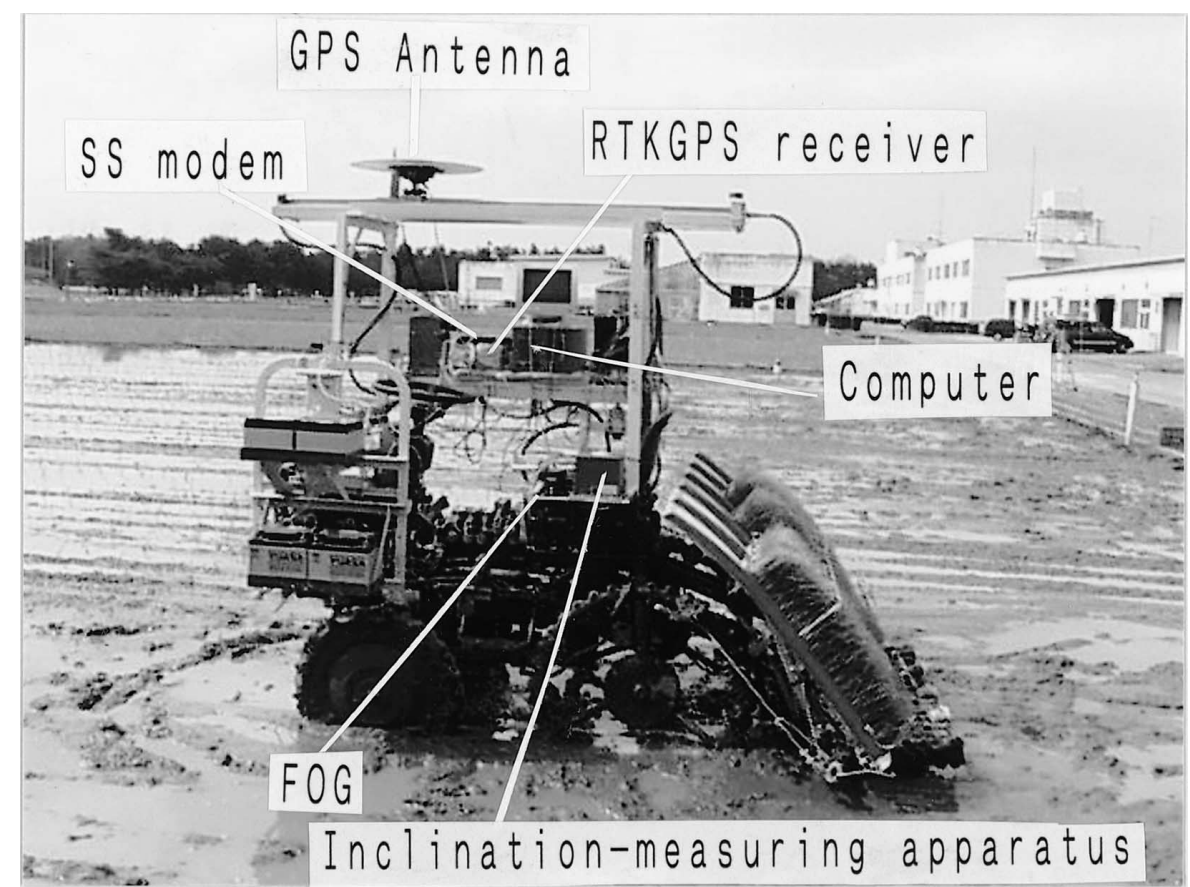

Fig. 1. Automated rice transplanter

as a rover station. Fig. 1 shows the modification of the rice transplanter for control by a computer whose CPU is Pentium $200 \mathrm{MHz}$. Fig. 2 shows the automated rice transplanting system. An RTKGPS with a $3 \mathrm{~cm}$ precision at $5 \mathrm{~Hz}$ data output was used to locate the position of the rice transplanter. For the communication between the reference station and the rover station, spread spectrum wireless modems were used. The baud rate was set at 19,200 bps. A FOG sensor was used to measure the yaw angle, and an inclination-measuring apparatus comprised of 3 FOG sensors and 3 accelerometers was used to mea- sure the roll and pitch angles. The steering, clutch, brake and transplanting instruments up-down were controlled by hydraulic valves. The engine throttle, CVT (Continuous Variable Transmission) lever, PTO clutch were controlled by DC-motors. The GPS data were transferred to a computer through $\mathrm{RS} 232 \mathrm{C}$ interface at $5 \mathrm{~Hz}$ sampling rate while other data were taken by the analog to digital converter board at $20 \mathrm{~Hz}$. The vehicle control data were transferred to hydraulic valves and DC-motor controllers by an analog to digital converter board and a digital I/O board.

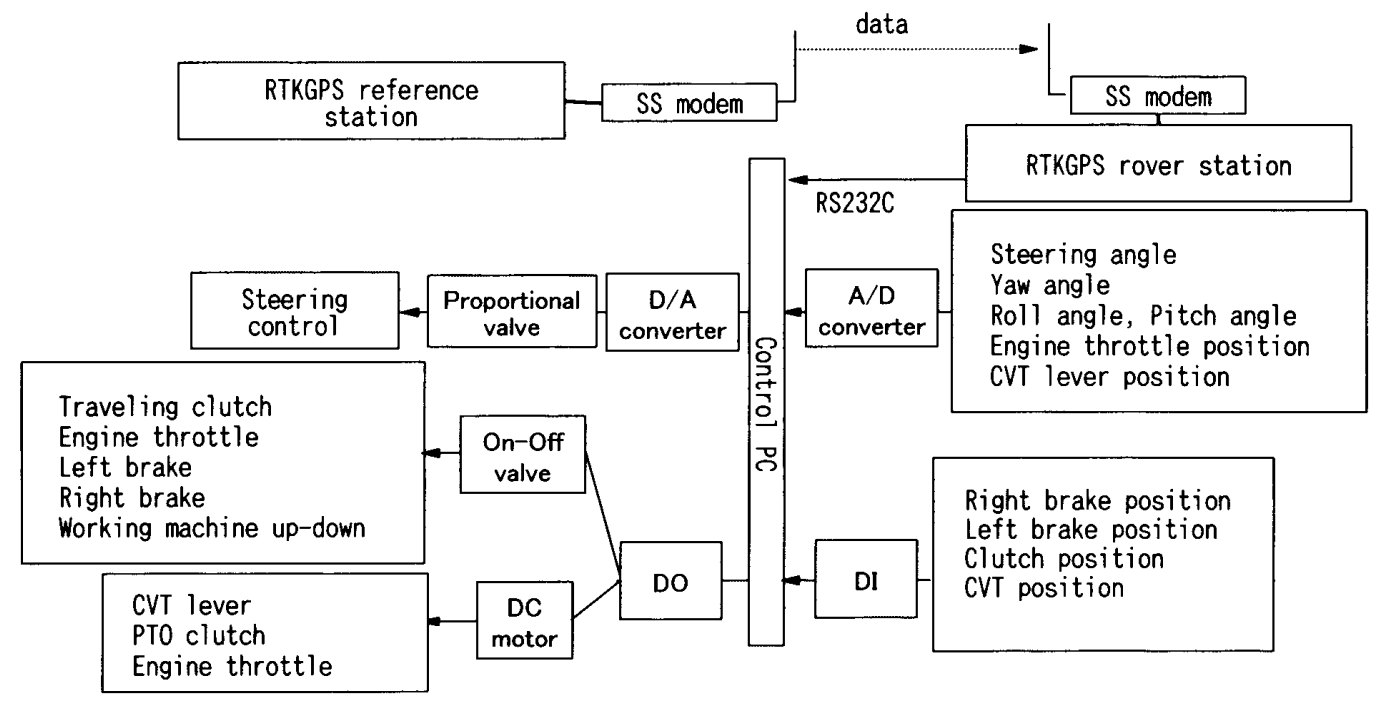

Fig. 2. Automated transplanting system 


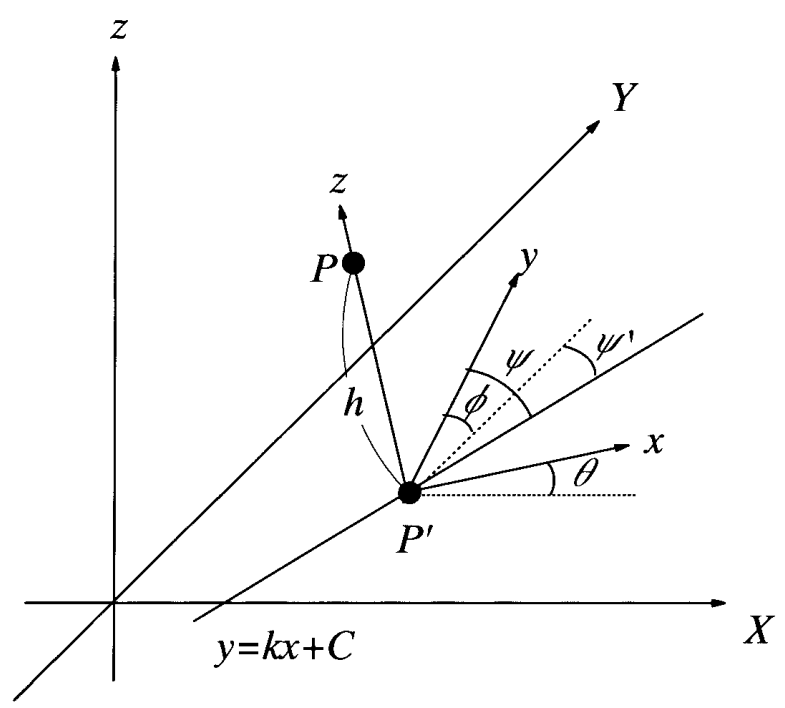

Fig. 3. Correction of the inclination

\section{2) Correction of the inclination}

When a rice transplanter travels in a paddy field, unevenness of the ground causes roll and pitch angles of about $3^{\circ}$. In this system, the GPS antenna is fixed on the rice transplanter. The GPS data indicate the position of the top of the GPS antenna. Assuming the antenna height is $2 \mathrm{~m}$, the horizontal distance between the top of the antenna and the bottom is $10.5 \mathrm{~cm}$ when the roll angle is $3^{\circ}$. To determine the precise position of the ground surface point under the GPS antenna, the influence of the roll and pitch angles must be corrected.

Fig. 3 shows the method of correction of the inclination. Assuming the presence of space coordinates $x y z$ on the rice transplanter and plane coordinates $X Y$ on which the rice transplanter travels, the straight desired path, $y=k X+C$ was derived on $X Y$. In this Figure it is assumed that the top of the GPS antenna corresponds to $P\left(p_{l}, q_{l}, h_{l}\right)$, the roll angle is $\theta$, the pitch angle is $\phi$, the yaw angle is $\psi$ and the GPS antenna height is $h$. The ground surface point under the GPS antenna $P^{\prime}$ is expressed as follows:

$$
\begin{array}{r}
P^{\prime}\left(p_{1}-\frac{h}{\sqrt{1+k^{\prime 2}}}\left(k^{\prime} \sin \theta+\sin \phi\right),\right. \\
q_{1}-\frac{h}{\sqrt{1+k^{\prime 2}}}\left(k^{\prime} \sin \phi-\sin \theta\right), \\
\left.h_{1}-h \cos \theta \cos \phi\right)
\end{array}
$$

In the above equation, the yaw angle $\mathrm{k}$ ' is expressed by the following equation,

$$
\begin{aligned}
\cos \psi^{\prime} & =\left(\sqrt{1+\left(\tan \psi \frac{\cos \theta}{\cos \phi}\right)^{2}}\right)^{-1} \\
k^{\prime} & =\frac{\sin \psi^{\prime}+k \cos \psi^{\prime}}{\cos \psi^{\prime}-k \sin \psi^{\prime}}
\end{aligned}
$$

3) Synchronization of the RTKGPS data, the yaw angle and the inclination data

The GPS rover station has to communicate with the reference station to obtain the reference data over a radio link. The rover receiver requires synchronized GPS measurement data from the reference station once per second. In this study, the authors used spread spectrum modems between reference and rover stations. Rover receiver's data output delay was about $0.2 \mathrm{~s}$. However, since the output intervals were not even, the time required to obtain the vehicle inclination had to be set to the time required to locate the vehicle position. Fig. 4 shows the flow of data sampling. The output of the RTKGPS receiver was 1 pulse per second (pps). In order to correct the time fluctuations, the authors used a pulse counter board in the computer. When 1 pps output was obtained on the board, the board started counting every $50 \mathrm{~ms}$. The yaw angle data were converted from analog to digital for every count, and the roll and pitch angles were converted for every 4 counts.

\section{4) Estimation of the real time deviation from the desired path}

To control the rice transplanter along the desired straight line, the real time deviation from the line must be estimated. The real time deviation $d$, was estimated by calculating the product of the speed and the yaw angle measured by the FOG sensor.

At $n$ seconds after the start, the deviation given by the vehicle speed and the yaw angle $d_{\mathrm{n}}$ was calculated by the following equation (2). In this equation (2), $v$ is the vehicle speed, $\psi(i)$ is the the yaw angle and $t_{s}$ is the sam-

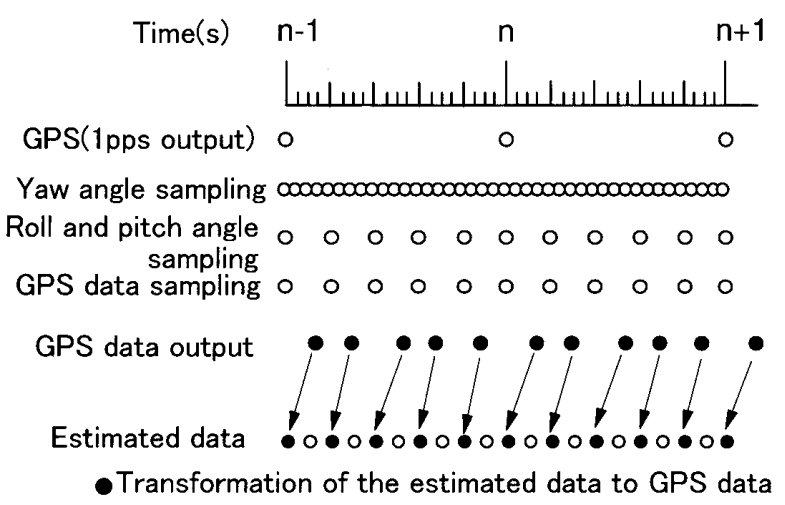

Fig. 4. Estimation of the real time deviation 
pling interval,

$$
d_{n}=\sum_{i=0}^{\frac{n}{t_{s}}} v t_{s} \psi(i)
$$

The deviation calculated by GPS data at $n$ seconds after the start was $d_{n G P S}$. $d_{n G P S}$ included the time delay $t_{d}$. At $n-t_{d}$ seconds after the start, the deviation given by the vehicle speed and the yaw angle $d_{n-t_{d}}$, was calculated by the following equation (3),

$$
d_{n-t_{d}}=\sum_{i=0}^{\frac{n-t_{d}}{t_{s}}} v t_{s} \psi(i)
$$

The estimated deviation $d_{n}$ included an error because the FOG sensor can not sense the side slip. Therefore, the difference between $d_{n-t_{d}}$ and $d_{n}$ was added to $d_{n G P S}$ to estimate the real time deviation with a small error,

$$
d=d_{n}-d_{n-t_{d}}+d_{n G P S}
$$

\section{5) Control method}

\section{(1) Straight drive}

The rice transplanter must be driven along the desired path. The deviation from the desired path is $d$. The aimed steering angle $\delta_{\text {aim }}$ and steering speed $\dot{\delta}$ were given by the following equations (5). In these equations, $\delta$ is the steering angle, $\psi$ is the yaw angle and $K_{p l}, K_{p 2}$ and $K_{p 3}$ are constant experimental data.

$$
\begin{aligned}
\delta_{\text {aim }} & =K_{p 1} d+K_{p 2} \psi \\
\dot{\delta} & =K_{p 3}\left(\delta-\delta_{\text {aim }}\right)
\end{aligned}
$$

\section{(2) Turn control}

Fig. 5 depicts the turning method. At interval A, the rice transplanter traveled along the desired path. When the vehicle reached the area located at $2.5 \mathrm{~m}$ from the levee, the traveling and working clutch was released.

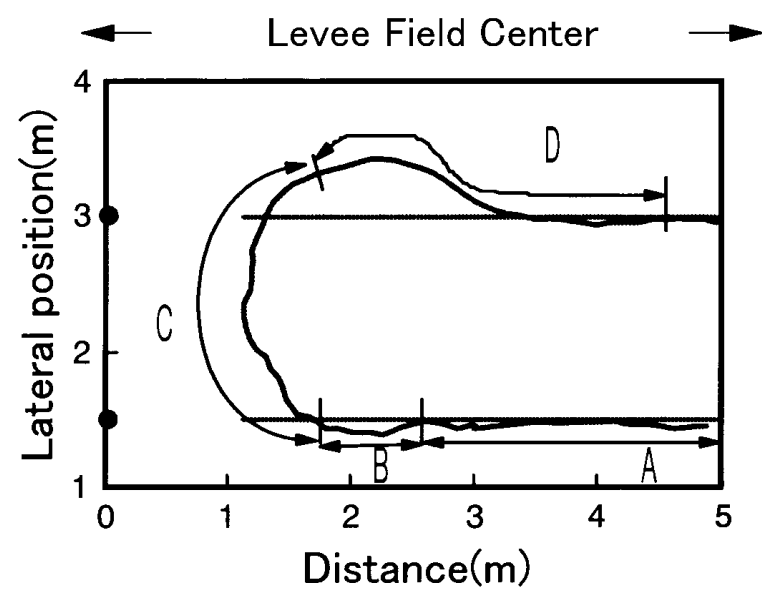

Fig. 5. Turn control
Then at interval B, the vehicle moved straight ahead over $0.7 \mathrm{~m}$, and at interval $\mathrm{C}$, the steering angle was set at $40^{\circ}$ and the brake was held. While the rice transplanter was turning, only the yaw angle data were used for the machine control. When the yaw angle was above $160^{\circ}$, the brake was released, and at interval $\mathrm{D}$, the steering angle was controlled by using equation (5).

\section{Results and discussion}

The experiment was conducted 3 days after puddling. Fig. 6 shows the deviation from the desired path, the steering angle and the yaw angle when the rice transplanter traveled straight at $0.8 \mathrm{~m} / \mathrm{s}$. The deviation from the desired straight path was less than $10 \mathrm{~cm}$ and the yaw angle changed by less than $3^{\circ}$. It is considered that the unevenness of the paddy terrain disturbed the direction of the rice transplanter. When the steering angle was set to get back close to the desired path, the paddy terrain

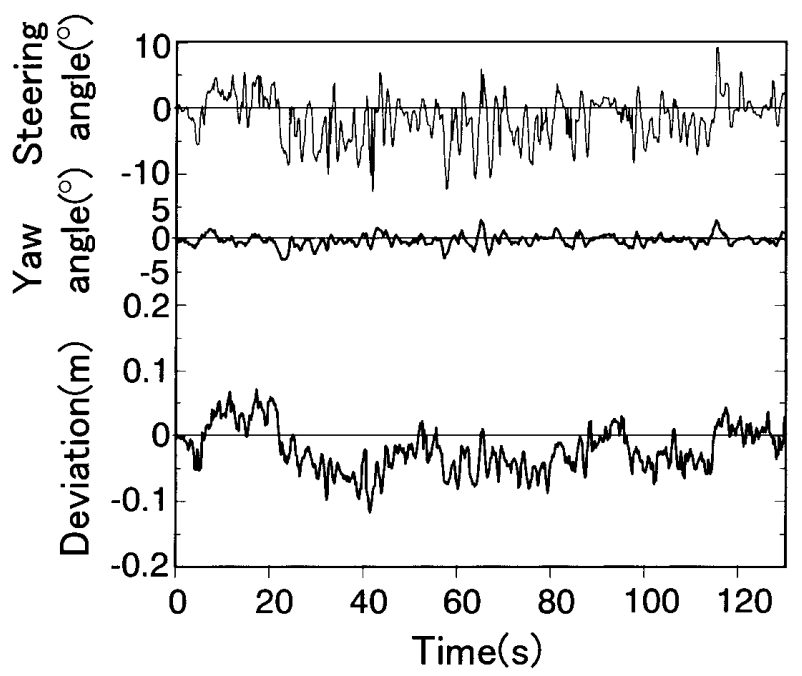

Fig. 6. Deviation from the desired path, yaw angle and steering angle in straight traveling

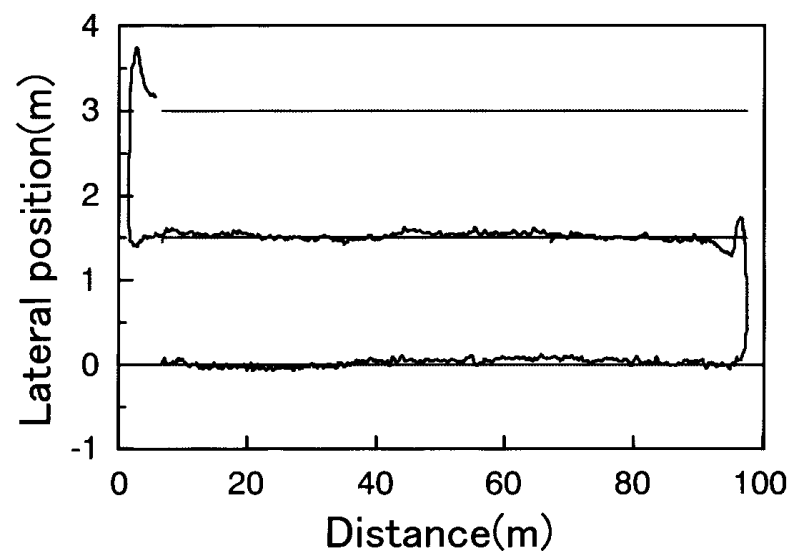

Fig. 7. Path of rice transplanting operation 
sometimes prevented the direction of the rice transplanter from changing. Therefore, a deviation from the desired path occurred. Fig. 7 shows the path of automated operation. When the vehicle got back close to the next desired path at the headland, overshooting occurred. At this point, the steering angle was controlled by using the equation (5). It is considered that the steering control was delayed because the deviation from the desired path and the yaw angle changed rapidly. The deviation from the desired path at the operation starting point was about $20 \mathrm{~cm}$. To get back close to the desired path, the rice transplanter had to travel $2 \mathrm{~m}$ ahead. It is necessary to go ahead and back at the headland when the vehicle turns at the headland to enhance the precision of operations.

\section{Conclusion}

In this study, a rice transplanter was driven automatically in a paddy field using a fiber optical gyro sensor and real time kinematic GPS. In this experiment, the influence of the vehicle inclination and the GPS time delay was corrected and the rice transplanter was well controlled. When the rice transplanter traveled over a distance of $100 \mathrm{~m}$ in a paddy field, the deviation from the target line was less than $10 \mathrm{~cm}$ at $0.8 \mathrm{~m} / \mathrm{s}$. After turning at the headland, the deviation from the desired path at the operation starting point was about $20 \mathrm{~cm}$.

\section{References}

1) Elkaim, G. et al. (1997): System identification and robust control of farm vehicles using carrier phase differential GPS. (http://einstein.Stanford.edu/gps/).

2) Inoue, K. et al. (1997): Estimation of place of tractor and adaptive control method of autonomous tractor using INS and GPS. In Preprints of the International Workshop on Robotics and Automated Machinery for Bio-Productions, 27-36.

3) Nagasaka,Y. et al. (1997): Automated operation in paddy fields with a fiber optic gyro sensor and GPS. In Preprints of the International Workshop on Robotics and Automated Machinery for Bio-Productions, 21-26.

4) Nagasaka,Y. et al. (1999): The development of autonomous rice transplanter (Part1). J. Jpn. Soc. Agric. Machin. 61(6), 179-186 [In Japanese with English summary].

5) Noguchi, N., Ishii, K. \& Terao, H. (1997): Development of an agricultural mobile robot using a geomagnetic direction sensor and image sensors. J. Agric. Eng. Res. 67, 1-15.

6) Yukumoto O. \& Matsuo Y. (1995): Research on autonomous land vehicle for agriculture. Proc. Int. Symp. Autom. Robotics Bioprod. Process. No. 1, 41-48. 